Monteagudo-Fernández, J., Rodríguez-Pérez, R.A., Escribano-Miralles, A. \& RodríguezGarcía, A.M. (2020). Percepciones de los estudiantes de Educación Secundaria sobre la enseñanza de la historia, a través del uso de las TIC y recursos digitales. Revista Electrónica Interuniversitaria de Formación del Profesorado, 23(2), 67-79.

DOI: https://doi.org/10.6018/reifop.417611

\title{
Percepciones de los estudiantes de Educación Secundaria sobre la enseñanza de la historia, a través del uso de las TIC y recursos digitales
}

José Monteagudo-Fernández ${ }^{(1)}$, Raimundo A. Rodríguez-Pérez ${ }^{(1)}$, Ainoa Escribano-Miralles ${ }^{(1)}$, Antonio M. Rodríguez-García ${ }^{(2)}$

${ }^{(1)}$ Universidad de Murcia, ${ }^{(2)}$ Universidad de Granada

\section{Resumen}

El artículo analiza las percepciones de los estudiantes de Educación Secundaria Obligatoria (ESO) y Bachillerato sobre el uso de las Tecnologías de la Información y la Comunicación (TIC) en la enseñanza de la historia. La muestra participante fue de 467 estudiantes de Institutos de Educación Secundaria de la Región de Murcia. Este estudio forma parte de un proyecto de investigación de mayor envergadura, relativo a enseñanza de la historia e implantación de metodologías y recursos innovadores. Del cuestionario estructurado para la recogida de información, se han seleccionado los ítems relativos al uso de las TIC y a la visión de los discentes. Los resultados arrojan una persistencia del modelo tradicional de enseñanza, que no prioriza metodologías cooperativas y de indagación, en el cual las TIC siguen teniendo un papel subsidiario. Se conciben como un complemento, más esporádico

\section{Contacto:}

Raimundo A. Rodríguez Pérez, raimundorodriguez@um.es, Dpto. Didáctica de las Ciencias Matemáticas y Sociales. Facultad de Educación, Universidad de Murcia. Campus de Espinardo. 30100 Espinardo (Murcia).

Este trabajo es resultado de los proyectos de investigación: "Conceptos metodológicos y métodos activos de aprendizaje para la mejora de las competencias docentes del profesorado" (PGC2018094491-B-C33), subvencionado por el Ministerio de Ciencia, Innovación y Universidades; "Competencias docentes y métodos activos de aprendizaje. Una investigación evaluativa con el profesorado en formación de ciencias sociales" (20638/JLI/18), financiado por la Fundación Séneca. Agencia de Ciencia y Tecnología de la Región de Murcia; "El pensamiento geográfico e histórico del alumnado de Educación Primaria en la Región de Murcia: propuesta metodológica innovadora para una educación de calidad" (20874/PI/18), financiado por la Fundación Séneca. Agencia de Ciencia y Tecnología de la Región de Murcia. 
aún en Bachillerato que en ESO. Las conclusiones determinan que mientras se siga enseñando historia con una visión factual y memorística es difícil formar ciudadanos críticos, con destrezas a la hora de construir su propio aprendizaje. Aunque el alumnado sea nativo digital será difícil que usen los recursos digitales para adquirir habilidades o valores, pues sigue priorizándose la transmisión pasiva de discursos cerrados.

\title{
Palabras clave
}

Percepciones de los estudiantes; enseñanza de la historia; Educación Secundaria; TIC.

\section{Perceptions of Secondary Education students on the teaching of history, through the use of ICT and digital resources}

\begin{abstract}
The article analyzes the perceptions of students of Compulsory Secondary Education (ESO) and Baccalaureate about the use of Information and Communication Technologies (ICTs) in the teaching of history. The sample used was 467 students from public High Schools of the Region of Murcia and was part of a larger research, related to the teaching of history and the implementation of innovative methodologies and resources. The items related to the use of ICTs and their vision among students have been selected. The results show a persistence of the traditional teaching model, which does not prioritize cooperative and inquiry methodologies, in which ICTs continue to have a subsidiary role. They are a complement, more sporadic even in Baccalaureate than in ESO. While continuing to teach history with a factual and memorial vision, it is difficult to train critical citizens, with skills when it comes to building their own learning. Even if they are digital natives, it will be difficult for them to use digital resources to acquire skills or values, since passive transmission of closed speeches continues to be prioritized.
\end{abstract}

\section{Key words}

Student perceptions; history teaching; Secondary Education; ICT.

\section{Introducción}

A día de hoy es de sobra conocido el papel que ocupan las Tecnologías de la Información y la Comunicación (TIC) en la vida diaria de las personas. Como sostenía Marquès (2012), "están ahí, forman parte de la cultura tecnológica que nos rodea y con la que debemos convivir". Tanto es así que, para Ramírez y González (2016), las nuevas investigaciones sobre las TIC ya no han de centrarse en la influencia de estas en la sociedad, sino sobre qué se hace con ellas, sobre todo en el ámbito que aquí nos interesa, el educativo, donde, nuevamente citando a Marquès (2012), una de las principales funcionalidades de las TIC, y toda una serie de recursos digitales que llevan aparejadas (WebQuests, mapas digitales, software, bases de datos, juegos de simulación, estrategias de gamificación, etc.), reside en su uso didáctico para facilitar los procesos de enseñanza y aprendizaje.

En el terreno de las ciencias sociales, ya defendía López (2005) que las TIC también ayudaban al alumnado a desarrollar habilidades relacionadas con la investigación, el análisis 
y la comunicación de ideas. Concretamente, en el caso de la asignatura de Historia, este mismo autor sostenía que el trabajo con TIC y determinados recursos digitales permitía el acceso a fuentes históricas, la comunicación con otros centros educativos, el desarrollo de proyectos colaborativos y la construcción de líneas de tiempo, o diagramas de causa efecto.

Así las cosas, si, como defendía Hernàndez (2011), la finalidad de la didáctica de las ciencias sociales era analizar, diseñar e investigar acerca de las técnicas didácticas, de divulgación y comunicación de los contenidos históricos (entre otros), así como los procesos de comprensión de los mismos, las tecnologías suponían una oportunidad para el docente como generador de imágenes virtuales en el espacio y el tiempo, siendo una opinión bastante generalizada que la utilización de las TIC y el camino hacia un aula virtual era un valioso recurso en el aprendizaje de las ciencias sociales en todos los niveles educativos (Osset, 2004).

Varios han sido los estudios en el último decenio que han insistido en la necesidad de incluir las TIC en el aula para mejorar el proceso de enseñanza de las ciencias sociales. Algunos de dichos estudios tienen que ver con el empleo de imágenes virtuales y la interactividad que nos proporcionan estos medios (Hernàndez, 2011), la utilización de la multimedia expositiva (Rivero, 2011), la arqueología virtual como herramienta para ayudar a explicar la evolución de los paisajes culturales a través de las recreaciones 3D (López-Menchero y Ramiro, 2015), el uso de blogs en el aula de Historia (Sobrino, 2013), la digitalización de recursos patrimoniales mediante códigos QR (Romero y Rodríguez-García, 2017), la introducción de los videojuegos para la enseñanza de la historia (Iturriaga y Medel, 2017), la implementación del aprendizaje móvil (m-learning) para el aprendizaje del patrimonio y la arqueología (Colomer, 2016), o la última novedad de la realidad aumentada (López, Miralles y Maquilón, 2019). En el ámbito internacional, muchas son las investigaciones que tratan de diseñar, aplicar y evaluar programas o proyectos de aprendizaje. Un ejemplo es el estudio desarrollado por Sanger, Sivelrman y Kraybill (2015) que trata de implementar y evaluar programas en línea basados en museos, con el fin de aumentar la capacidad de los educadores de museos y los docentes para desarrollar colaboraciones en el aprendizaje y cultivar secuencias de aprendizaje profundas y colaborativas, así como potenciar en los docentes destrezas y conocimientos para aprovechar los recursos patrimoniales y fuentes históricas de los museos.

No obstante, y a pesar de las experiencias e investigaciones relatadas, Ramírez y González (2016) sostenían que la transferencia al proceso de enseñanza y aprendizaje no se apreciaba realmente en la mayoría de los centros escolares debido a las reticencias que tenía el profesorado hacia su uso, unas reservas motivadas fundamentalmente por la formación de los propios docentes, la variable que más incide en el nivel de competencia mediática (González, Gonzálvez y Ramírez, 2015). Ello hace que en la mayoría de ocasiones la labor del enseñante se quede, empleando palabras de Marquès (2012), en un escenario de aprender de las TIC y no se llegue a aprender con las TIC para así poder innovar en las prácticas docentes e introducir cambios reales en las prácticas cotidianas de las aulas que lleven a conseguir mejores aprendizajes (De Haro, 2009; Hernández y Martín, 2017).

En esta línea han apuntado recientes investigaciones sobre profesorado en formación, supuesto "nativo digital", tales como la de Rodríguez-García, Romero y Campos (2018) sobre el bajo nivel de competencia digital y de conocimiento del futuro profesorado de Educación Primaria acerca de recursos tecnológicos como MOOC y WebQuest, y al acceso a las plataformas en Internet que los alojan; las conclusiones del trabajo de Girón, Cózar y González-Calero (2019) sobre el escaso nivel de los estudiantes de los grados de maestro en la competencia digital al final de sus estudios; la función lúdica o motivacional que el futuro profesorado de Geografía e Historia de Secundaria concede a las TIC (Miralles, Gómez y 
Monteagudo, 2019) y a los recursos digitales, no permitiendo así un abordaje crítico que desarrolle en el alumnado la capacidad de evaluar información digital y resolver interrogantes históricos (Miralles, Gómez, Arias y Fontal, 2019).

De este modo, si todos los recursos y herramientas no son utilizados adecuadamente no pueden dar lugar a mejoras en la educación histórica (Miralles, 2011). La simple presencia de las TIC en los centros de enseñanza no garantiza la innovación, sino que son necesarios cambios en el rol del profesor, en la metodología y en el sistema de evaluación (Marquès, 2012; Romero, Castejón, López y Fraile, 2017). El uso de estos medios debe servir para fomentar el pensamiento autónomo, ético, creativo y crítico. La alfabetización digital, como cualquier otra, necesita ser enseñada y aprendida para ayudar al alumnado a pensar históricamente. Por tanto, no solamente se precisa un conocimiento técnico, sino también pedagógico (Cabero y Barroso, 2016).

Todo lo hasta ahora dicho presenta un cariz más ineludible en un campo de conocimiento como el de las ciencias sociales, cuya principal función es la formación de ciudadanía (Audigier, 1999). De ahí, nuestro interés por conocer las percepciones que el alumnado de Educación Secundaria de ciencias sociales tiene sobre el empleo de las TIC y los recursos digitales en el aula para la enseñanza de la historia. Ello es importante porque las respuestas del alumnado nos servirán para conocer aspectos relevantes de la metodología aplicada en el proceso de enseñanza-aprendizaje de la historia, y más en una Comunidad Autónoma como la Región de Murcia, que cuenta con uno de los porcentajes más bajos de dominio de la competencia mediática según el estudio que realizaron González, Gonzálvez y Ramírez (2015).

\section{Objetivos}

El objetivo principal de la investigación ha sido el de conocer las percepciones del alumnado de Educación Secundaria respecto al uso de las TIC y otros recursos digitales en la enseñanza de la historia. Para ello se concretaron otros tres objetivos específicos:

1. Averiguar la presencia de las TIC y los recursos digitales en la metodología docente de las clases de Historia.

2. Conocer qué incidencia tiene el uso de las TIC y los recursos digitales sobre la motivación del alumnado en las clases de Historia.

3. Saber los efectos que presenta el uso de las TIC y los recursos digitales en el aprendizaje y la transferencia del conocimiento histórico del alumnado.

\section{Metodología}

\section{Participantes}

La población objeto de estudio estuvo compuesta por los estudiantes de Educación Secundaria Obligatoria y Bachillerato de la Región de Murcia que cursaban asignaturas de Geografía e Historia en IES públicos de dicha Comunidad Autónoma. En este caso, la elección de los grupos discentes estuvo ligada a la asignación de los centros de prácticas curriculares al alumnado de la especialidad de Geografía e Historia del Máster en Formación del Profesorado de la Universidad de Murcia durante el curso 2018/2019.

Finalmente, los participantes fueron 467 estudiantes, de los cuales 222 eran hombres $(47,54 \%)$ y 245 mujeres $(52,46 \%)$. La mayoría de estos alumnos tenía entre doce y diecisiete 
años (96\%). En torno a las características de los participantes, 385 alumnos (82,44\%) no habían repetido nunca, frente a 82 (17,56\%) que sí lo habían hecho alguna vez, fundamentalmente en la ESO (70,7\%) y Primaria (23,17\%).

En cuanto a su distribución por etapas, 375 alumnos cursaban ESO (80,3\%), siendo más numerosos los estudiantes del primer (150 alumnos 32,12\%) y cuarto curso (125 alumnos $26,76 \%) ;$ y 92 (19,75\%) de Bachillerato, donde destacaban los 76 discentes de primer año $(16,27 \%)$. En total, se recogieron datos de 18 grupos de 14 centros, 13 públicos y 1 privado, en 9 localidades diferentes de la Región, tanto a nivel socioeconómico como geográfico.

\section{Diseño}

El diseño empleado en la presente investigación ha sido un diseño cuantitativo no experimental tipo encuesta y trasversal. Se ha optado por este tipo de enfoque metodológico debido a que se trata de un método de investigación capaz de dar respuesta a problemas tanto en términos descriptivos como de relación de variables cuando la información es recogida de forma sistemática, garantizando el rigor de los datos obtenidos (Buendía, Colás y Hernández-Pina, 1998). Tal y como señalan Hernández-Pina y Maquilón (2010), los diseños mediante encuesta son muy habituales en el ámbito de la educación ya que son aplicables a múltiples problemas y permiten recoger información sobre un número elevado de variables.

\section{Instrumento}

Para la recogida de información se aplicó un cuestionario dividido en cuatro bloques, el cual se denominó "Valoración del alumnado de Secundaria sobre la enseñanza de la Historia". El primer bloque del cuestionario estaba centrado en conocer la metodología didáctica empleada en las clases de Historia en Educación Secundaria. El segundo, indagaba sobre la motivación que despertaba en el alumnado las clases de Historia. El tercero, buscaba conocer la satisfacción del alumnado por las clases de Historia. Finalmente, en el cuarto bloque se examinaban las percepciones del alumnado respecto al aprendizaje y la transferencia del conocimiento adquirido en las clases de Historia. El cuestionario contaba con una escala tipo Likert de 5 valores para medir el grado de acuerdo y desacuerdo, siendo 1 Muy en desacuerdo, y 5 Muy de acuerdo.

Siguiendo el procedimiento general para la construcción y aplicación de instrumentos cuantitativos de recogida de información (McMillan y Schumacher, 2005), se diseñó una amplia batería de ítems, hasta un total de 40. Configurada una primera versión del cuestionario, se procedió a la validez de contenido a través de procedimiento inter jueces en torno a las categorías de pertinencia y claridad de los ítems del instrumento. Para esta validación del contenido se optó por un grupo de discusión con siete expertos, tres profesores del área de Didáctica de las Ciencias Sociales, dos profesores de Geografía e Historia de Educación Secundaria, y dos profesores de Ciencias Sociales de Educación Primaria. Se optó por el método Delphi y, tras las modificaciones pertinentes, se realizó una segunda ronda con los expertos para validar definitivamente el instrumento. Todas las recomendaciones recibidas fueron consideradas, dando como resultado el cuestionario final.

Antes de proceder al análisis de datos se estimó el grado de fiabilidad y validez del constructo del test. Para analizar la fiabilidad del cuestionario se empleó el método de consistencia interna basado en el Alfa de Cronbach, que permite estimar la fiabilidad de un instrumento de medida compuesto por un conjunto de ítems, de tipo escala Likert, que esperamos midan la misma dimensión teórica (el mismo constructo). Este procedimiento de validación ha sido utilizado también por otros trabajos de educación histórica 
(Gestsdóttir, Van Boxtel y Van Drie, 2018). El criterio establecido y señalado por diferentes autores es que un valor del Alpha de Cronbach, entre 70 - .90, indica una buena consistencia interna (González y Pazmiño, 2015; Oviedo y Campo-Arias, 2005). El análisis de fiabilidad ha obtenido un Alpha de Cronbach igual a (.913), indicando una elevada consistencia interna. Igualmente se procedió al análisis del coeficiente de dos mitades de Guttman con un resultado satisfactorio $(, 826)$.

También se comprobó la validez del constructo y la viabilidad de un posterior análisis factorial. Para ello se analizó la matriz de correlaciones y se realizó la Prueba de Esfericidad de Barlett, resultando significativa $(p<.001)$, con un valor de KMO de .914. Estos datos mostraron que la matriz del instrumento es adecuada para ser factorizada.

\section{Procedimiento de análisis de la información}

Los datos recogidos se codificaron y analizaron en el programa estadístico R-Studio versión 3.6.1. Para esta investigación se realizaron análisis de estadísticos descriptivos, tanto de los ítems de forma individual como de su relación con el resto de ítems de cada bloque (mínimo, máximo, media y mediana, así como el porcentaje de las diferentes respuestas), e inferencial para buscar diferencias significativas en los ítems del cuestionario según las diferentes variables socio-demográficas. Para ello, se utilizaron los tests no-paramétricos de la $U$ de Mann-Whitney para factores de dos niveles y la $K$ de Kruskal-Wallis para factores de tres o más niveles.

\section{Resultados}

En relación al primer bloque del cuestionario, relacionado con la metodología seguida por el profesorado para la enseñanza de la historia, se puede afirmar que se trata de una metodología tradicional en la que predomina la explicación del docente en base al manual de la asignatura y en la que, a pesar del empleo de diferentes instrumentos de evaluación, el examen escrito es la principal herramienta para valorar el aprendizaje del alumnado, un aprendizaje que es, en esencia, memorístico. Sorprende el bajo nivel de uso de documentos históricos, visitas didácticas o trabajos grupales. En lo que respecta al uso de Internet (Figura 1), un tercio del alumnado manifiesta no haberlo empleado nunca (13,20\%) o casi nunca (19,70\%), mientras que un $26,3 \%$ alega usar Internet para trabajar historia algunas veces. El 40,80\% restante dice usarlo siempre o casi siempre a partes iguales. Nos encontramos, pues, ante un uso moderado de las nuevas tecnologías en el proceso de enseñanza-aprendizaje de la historia.



Figura 1. Respuestas y porcentaje del ítem 9

En el análisis inferencial por sexos se encontraron diferencias significativas con $W=30109$ ( $p$ $<0.05$ ). Fueron los varones quienes mostraron una mayor percepción en el uso de las TIC en las clases de Historia. Tratándose de los mismos grupos y contenidos trabajados, podemos inferir que las chicas tienen una idea distinta de lo que supone trabajar con las TIC. 
También hallamos diferencias significativas en las variables de "Etapa" ( $W=26610.5, p<$ $0.05)$ y "Curso" $(\chi 2(5)=78.81, p<0.05)$. Es el alumnado de ESO quien ofrece mayor cantidad de respuestas positivas al uso de las TIC en las clases de Historia, muy por encima del de Bachillerato, sobre todo en segundo curso.

Asimismo, existen diferencias significativas en la variable "Repetidor" ( $W=12710.5, p<0.05)$. Es el alumnado que ha repetido alguna vez en el sistema educativo el que arroja respuestas más negativas en lo que al empleo de las TIC en el aula para trabajar contenidos históricos se refiere. Volvemos a encontrar una percepción diferente a la del resto de sus compañeros habiendo trabajado los mismos contenidos en el mismo grupo con los mismos docentes.

El bloque 2 del cuestionario, relacionado con la motivación que genera en el alumnado las clases de Historia, se comprueba que los discentes no están de acuerdo mayoritariamente $(57,1 \%)$ con que las clases de Historia les motive porque trabajan en grupo (como se ha apuntado anteriormente), o solamente por el mero hecho de aprobar la asignatura (51,1\%), sino porque consideran que comprenden mejor la realidad en la que se desenvuelven $(49,8 \%)$ y por los recursos que emplean en el aula diferentes al libro de texto, como se observa en la figura 2 , entre los que se encuentra Internet (46,8\%). Podemos ver que, a pesar del escaso uso, el empleo de las TIC motiva y debería aprovecharse para utilizarse más.

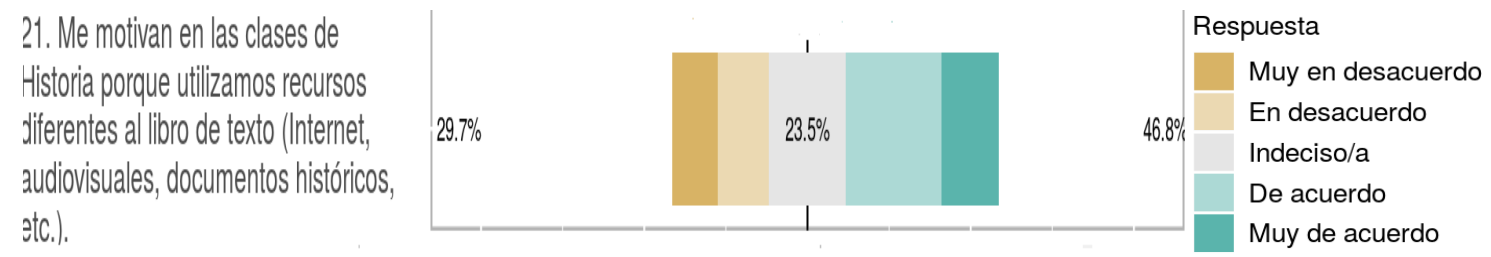

Figura 2. Respuestas y porcentaje del ítem 21

En relación a este ítem número 21 , volvieron a encontrarse diferencias significativas en "Etapa" $(W=25634.5, p<0.05)$ y "Curso" $(\chi 2(5)=68.9, p<0.05)$. Es nuevamente el alumnado de ESO el que ofrece mayores respuestas positivas ante la motivación que le despierta el trabajo con TIC respecto a sus compañeros de Bachillerato, pero ya no de segundo curso, sino de primero, donde se obtiene el mayor porcentaje de respuestas negativas. Nos encontramos aquí una realidad destacada: si bien el alumnado de $20^{\circ}$ de Bachillerato expresa un menor uso de las TIC en las clases de Historia, le motiva más dicho uso, aunque sea escaso.

El tercer bloque no contaba con ningún ítem relacionado con el uso de las TIC en el aula de Historia, pero sí el cuarto y último (ítem 38), ligado a la percepción del aprendizaje y la transferencia del conocimiento. En este sentido, los estudiantes manifiestan, de manera mayoritaria, aprender los principales hechos $(71,9 \%)$ y personajes históricos $(66,4 \%)$, así como los cambios y continuidades en la historia (62,2\%), y las causas y consecuencias de los sucesos históricos $(60,6 \%)$. En el polo opuesto, y con la puntuación desfavorable más elevada (44\%), el alumnado considera que no aprende diferentes formas de usar las TIC en las clases de Historia, frente a un $29,7 \%$ de indecisos y un $26,3 \%$ que se muestra de acuerdo (Figura 3). 




Figura 3. Respuestas y porcentaje del ítem 38

En este ítem volvieron a encontrarse diferencias significativas en relación con la etapa ( $W=$ $23229.5, p<0.05)$ y curso $(\chi 2(5)=50.03, p<0.05)$. Es de nuevo el alumnado de ESO el que ofrece menores respuestas negativas ante la motivación que le despierta el trabajo con TIC en las clases de Historia respecto a sus compañeros de Bachillerato, sobre todo de primer curso. El parco uso y motivación que generan las TIC en la etapa de Bachillerato se plasma en las respuestas del alumnado sobre el escaso aprendizaje que logra con el uso de las TIC.

\section{Discusión y conclusiones}

Los ítems analizados sobre TIC y recursos digitales muestran una fotografía fija y, por ende, recurrente en la enseñanza de la historia en Educación Secundaria Obligatoria y Bachillerato. La huella indeleble de reformas legislativas involucionistas, así como las inercias docentes impiden una presencia habitual de las TIC en las clases de Historia. Y cuando se da su uso es complementario, cuasi accidental, por lo general. La necesidad de concluir temarios enciclopédicos y culturalistas, sobrecargados de contenidos conceptuales, y de evaluar de forma burocratizada impide salir de la trilogía tradicional: lección magistral, manual y examen escrito de tipo memorístico. Sobre esto ya advirtieron los trabajos de Merchán (2005 y 2007) y Domínguez (2015). Cambia la ley, cambia la sociedad, se modernizan los recursos digitales, pero la forma de impartir historia apenas se modifica.

Las percepciones de los estudiantes arrojan resultados según los cuales, en el salto de una etapa a otra, es decir de ESO a Bachillerato, esta última denota menor relevancia de las TIC. Ello supone un cambio en la metodología docente en lo que al uso de las TIC concierne para enseñar historia. Quizá la EBAU, con su enorme carga conceptual, determina una enseñanza aún más tradicional, poco proclive al empleo de las nuevas tecnologías en el aula. No obstante, aunque sea más infrecuente su uso, la percepción de los estudiantes de $2 .^{\circ}$ de Bachillerato sobre las TIC resulta más positiva que entre los discentes de ESO. Ello puede deberse a que el empleo de herramientas distintas al libro de texto y la explicación docente alienta más a los discentes, en un curso de calendario especialmente intenso, por ser preparatorio para el acceso a la universidad. Por tanto, salir de la lección magistral y el manual supone un excurso reparador. En cualquier caso, la visión más negativa del uso de las TIC viene de los estudiantes repetidores, algo que puede estar relacionado con su situación académica, peor que la del resto de sus compañeros.

La función de esta materia sigue centrada en hacer un amplio repaso de la historia universal y española, con una óptica esencialmente europea (López Facal, 2010), frente a la visión española de Educación Primaria. La sucesión de culturas, civilizaciones, imperios y regímenes políticos sigue siendo hegemónica, máxime en Bachillerato, sobre todo en su segundo curso, preparatorio para la prueba de acceso a la universidad. Una historia que, siendo interesante, deviene en algo anodino en tanto que estático, no interpela al estudiante ni a su vida cotidiana. Se aprende como algo exógeno, que no permite siquiera crítica o desmontar tópicos sobre la historia tradicional (López Facal, Miralles, Prats y 
Gómez, 2017). Si bien es cierto que, frente a la visión esencialista de antaño, forjadora de una identidad patriótica, a partir del último tercio del siglo XX, la historia ha virado hacia una enseñanza edulcorada, que esquiva tabúes sobre memoria histórica, minorías, represaliados y grupos sociales marginados (Valls, 2007).

En cualquier caso, a pesar de la inevitable evolución, ligada a la modernización de España y su plena inserción en las instituciones europeas, la metodología y recursos con que se enseña historia apenas han cambiado. Pueden mutar ciertos formatos, pero no son transformaciones sustanciales. Mientras que en Educación Infantil y Educación Primaria abundan los centros que trabajan por proyectos, centros de interés y otras metodologías cooperativas y de indagación (Galindo, 2016), a partir de ESO se vuelve harto infrecuente (Gómez, Ortuño y Miralles, 2018). A mayor nivel educativo, mayor cantidad de saberes de primer orden: conceptos, fechas, datos, hechos. Siendo estos necesarios, toda vez que suponen un cimiento básico para construir aprendizajes cooperativos, destrezas y habilidades, su predominio es tal que cuesta salir de esos relatos cerrados sobre: política interior y exterior, economía, sociedad y cultura (Cuenca, Estepa y Martín, 2017; Rodríguez y Solé, 2018). Así lo recuerdan cuando llegan a la universidad (Gómez, Rodríguez y Mirete, 2018). Frente a otros países donde los retos o inicios a la investigación histórica son una constante en todas las etapas educativas (Gómez, Miralles y Chapman, 2017), desde los primeros niveles, en España eso se antoja un desiderátum (Prats, 2011).

Las TIC podrían ayudar a realizar esa necesaria evolución, pero no por ello deben ser el único recurso, sino un instrumento, un auxiliar necesario. Actualmente, predomina su uso, y así se recoge en la muestra analizada, como algo lúdico y ocasional (Cózar, 2017). El paso siguiente debería ser ir de las TIC a las TAC (Tecnologías del Aprendizaje y el Conocimiento), que ayuden al discente a resolver problemas e interrogantes útiles en su vida cotidiana, tanto en entornos y épocas recientes como lejanas. Para llegar, finalmente, a la TEP (Tecnologías del Empoderamiento y la Participación), aquellas que fomenten un espíritu cívico y crítico (Cabero, Torres y Hermosilla, 2019), es decir ciudadanos concienciados con las desigualdades sociales, el medio ambiente y el patrimonio (Cambil y Romero, 2016). No en vano, el uso adecuado de las TIC puede contribuir al empoderamiento tanto de docentes como de discentes (Castañeda, Esteve y Adell, 2018). Memorizando reinados y guerras esto seguirá siendo una entelequia. La selección de temas y su abordaje en profundidad, dentro y fuera de las aulas, puede ser un revulsivo. Las TIC están llamadas a jugar un papel decisivo, dado que facilitan el acceso a una enorme cantidad de información, permiten trabajos colaborativos y construcción del propio conocimiento. Se trata, pues, de construir los aprendizajes de los discentes -rol activo- y no limitarse a asimilar verdades inmutables ajenas -rol pasivo-.

A pesar del enorme peso de lo tradicional, los estudiantes que formaron parte de la muestra analizada comprenden la utilidad de la historia, al ser una materia transversal sobre el ser humano y las sociedades. Sin embargo, las TIC difícilmente casan con una enseñanza de ese tipo, poco afín a la innovación educativa, en tanto tendente reforzar identidades (López Facal, 2014). La innovación -que existe, pero no es mayoritaria- no vendrá de la mano del uso de las TIC, sino de que esto suponga un cambio real, que conceda protagonismo a los discentes y les transforme en ciudadanos críticos, que adquieren valores cívicos y democráticos. Ello supera el interés de cualquier recurso didáctico, incluidas las TIC, pero sin ellas resulta inviable. 


\section{Agradecimientos}

Los autores agradecen la realización del análisis estadístico a la Sección de Apoyo Estadístico (SAE), Área Científica y de Investigación (ACTI), Universidad de Murcia (www.um.es/web/acti).

\section{Referencias}

Audigier, F. (1999). L'éducation à la citoyenneté. París: INRP.

Buendía, L., Colás, P. y Hernández-Pina, F. (1998). Métodos de investigación en psicopedagogía. Madrid: McGraw-Hill.

Cabero, J., y Barroso, J. (2016). ICT teacher training: a view of the TPACK model/Formación del profesorado en TIC: una visión del modelo TPACK. Cultura y educación, 28(3), 633-663.

Cabero, J., Torres, L. y Hermosilla, J.M. (2019). Las TIC y la creación de una ciudadanía crítica e-digital. Education in the knowledge society (EKS), 20.

doi: http://dx.doi.org/10.14201/eks2019_20_a22

Cambil, M.E. y Romero. G. (2016). Las TIC, las TAC y Redes 3.0 para la enseñanza de las Ciencias Sociales. En Á. Liceras y G. Romero (Coords.), Didáctica de las ciencias sociales. Fundamentos, contextos y propuestas (pp. 271-295). Madrid: Pirámide.

Castañeda, L., Esteve, F. y Adell, J. (2018). ¿Por qué es necesario repensar la competencia docente para el mundo digital? RED. Revista de Educación a Distancia, 56. Recuperado de http://www.um.es/ead/red/56/castaneda_et_al.pdf

Colomer, J.C. (2016). Historypin: una app para el trabajo del pensamiento histórico en Didáctica de las Ciencias Sociales. En VV.AA. (Coords.), IV Congreso Internacional de Investigación en Innovación Educativa (pp. 171-176). Murcia: Editum.

Cózar, R. (2017). Usos y abusos de las TIC en la enseñanza de la historia. En P. Sanz, J. Molero y D. Rodríguez (Eds.), La historia en el aula. Innovación docente y enseñanza de la historia en la educación secundaria (pp. 211-242). Lleida: Milenio.

Cuenca, J.M., Estepa, J. y Martín, M.J. (2017). Patrimonio, educación, identidad y ciudadanía. Profesorado y libros de texto en la enseñanza obligatoria. Revista de Educación, 375, 136-159.

De Haro, J.J. (2009). Algunas experiencias de innovación educativa. Arbor: Ciencia, pensamiento y cultura, CLXXXV, 71-92. doi: 10.3989/arbor.2009.extran1207

Domínguez, J. (2015). Pensamiento histórico y evaluación de competencias. Barcelona: Graó.

Gestsdóttir, S.M., Van Boxtel, C., y Van Drie, J. (2018). Teaching historical thinking and reasoning: Construction of an observation instrument. British Educational Research Journal, 44(6), 960-981. doi: 10.1002/berj.3471

Galindo, R. (2016). Enseñar y aprender Ciencias Sociales en Educación Primaria. Modelo didáctico y estrategias metodológicas. En Á. Liceras y G. Romero (Coords.), Didáctica de las Ciencias Sociales. Fundamentos, contextos y propuestas (pp. 73-94). Madrid: Pirámide. 
Girón, V., Cózar, R. y González Calero, J.A. (2019). Análisis de la autopercepción sobre el nivel de competencia digital docente en la formación inicial de maestros/as. Revista Electrónica Interuniversitaria de Formación del Profesorado, 22(3), 193-218.

Gómez, C.J., Miralles, P. y Chapman, A. (2017). Los procedimientos de evaluación en la clase de Historia. Un análisis comparativo a través de las opiniones de los docentes en formación en España e Inglaterra. Revista Electrónica Interuniversitaria de Formación del Profesorado, 20(2), 45-61.

Gómez, C.J., Ortuño, J. y Miralles, P. (2018). Enseñar ciencias sociales con métodos activos de aprendizaje. Reflexiones y propuestas a través de la indagación. Barcelona: Octaedro.

Gómez, C.J., Rodríguez, R.A., y Mirete, A.B. (2018). Percepción de la enseñanza de la historia y concepciones epistemológicas. Una investigación con futuros maestros. Revista Complutense de Educación, 29(1), 237-250.

González, N., Gozálvez, V. y Ramírez, A. (2015). La competencia mediática en el profesorado no universitario. Diagnóstico y propuestas formativas. Revista de Educación, 367, 117146. doi: 10.4438/1988-592X-RE-2015-367-284

González, J. y Pazmiño, M. (2015). Cálculo e interpretación del Alfa de Cronbach para el caso de validación de la consistencia interna de un cuestionario, con dos posibles escalas tipo Likert. Revista Publicando, 2(1), 62-77.

Hernàndez, F.X. (2011). La iconografía en la didáctica de las ciencias sociales. Íber. Didáctica de las Ciencias Sociales, Geografía e Historia, 68, 7-16.

Hernández, A. y Martín, J. (2017). Concepciones de los docentes no universitarios sobre el aprendizaje colaborativo con TIC. Educación XX1, 20(1), 185-208. doi: 10.5944/ educXX1.14473

Hernández-Pina, F. y Maquilón, J.J. (2010). Introducción a los diseños de investigación educativa. En S. Nieto (Ed.), Principios, métodos y técnicas esenciales para la investigación educativa (pp.109-126). Madrid: Dykinson.

Iturriaga, D. y Medel, I. (2017). La historia a través de los videojuegos. Íber. Didáctica de las Ciencias Sociales, Geografía e Historia, 86, 30-36.

López, A., Miralles, P. y Maquilón, J.J. (2019). Design, Application and Effectiveness of an Innovative Augmented Reality Teaching Proposal through 3P Model. Applied Sciences, 9, 5426. doi: 10.3390/app9245426

López Facal, R. (2010). Nacionalismos y europeísmos en los libros de texto: Identificación e identidad nacional. Clío \& Asociados: la historia enseñada, 14, 9-33.

López Facal, R. (2014). La LOMCE y la competencia histórica. Ayer, 74, 273-285.

López Facal, R., Miralles, P., Prats, J. (Dirs.) y Gómez, C.J. (Coord.) (2017). Enseñanza de la historia y competencias educativas. Barcelona: Graó.

López, J. C. (2005). Integración de las TIC en Ciencias Sociales. Eduteka. Recuperado de http://www.eduteka.org/articulos/Editorial21

López-Menchero, V.M. y Ramiro, R. (2015). La arqueología virtual como recurso para la comprensión del paisaje cultural. íber. Didáctica de las Ciencias Sociales, Geografía e Historia, 81, 41-44.

Marquès, P. (2012). Impacto de las TIC en la educación: funciones y limitaciones. 3 C TIC: cuadernos de desarrollo aplicados a las TIC, 2(1), 2-15. 
McMillan, J.H. y Schumacher, S. (2005). Investigación educativa. Madrid: Pearson.

Merchán, F.J. (2005). Enseñanza, examen y control: profesores y alumnos en la clase de Historia. Barcelona: Octaedro.

Merchán, F.J. (2007). El papel de los alumnos en la clase de Historia como agentes de la práctica de la enseñanza. Didáctica de las Ciencias Experimentales y Sociales, 21, 33-51.

Miralles, P. (2011). La historia y el uso de las nuevas tecnologías de la información y la comunicación. En Enseñanza y aprendizaje de la Historia en la Educación Básica (pp. 126-142). México: Secretaría de Educación Pública y Universidad Pedagógica Nacional de México.

Miralles, P., Gómez, C.J., Arias, V.B. y Fontal, O. (2019). Recursos digitales y metodología didáctica en la formación inicial de docentes de Historia. Comunicar: Revista Científica de Comunicación y Educación, 61, 45-56. doi: 10.3916/C61-2019-04

Miralles, P., Gómez, C.J. y Monteagudo, J. (2019). Percepciones sobre el uso de recursos TIC y «mass-media» para la enseñanza de la historia. Un estudio comparativo en futuros docentes de España-Inglaterra. Educación XX1, 22(2), 187-211. doi: 10.5944/educXX1.21377

Osset, J. (2004). Las nuevas tecnologías en ciencias sociales. Un viaje hacia el aula virtual. Íber. Didáctica de las Ciencias Sociales, geografía e historia, 4, 57-67.

Oviedo, H.C. y Campo-Arias, A. (2005). Aproximación al uso del coeficiente alfa de Cronbach. Revista Colombiana de Psiquiatría, 34(4), 572-580.

Prats, J. (Coord.) (2011). Geografía e Historia. Investigación, innovación y buenas prácticas. Barcelona: Graó.

Ramírez, A. y González, N. (2016). Competencia mediática del profesorado y del alumnado de educación obligatoria en España. Comunicar: Revista científica iberoamericana de comunicación y educación, 24(49), 49-58. doi: 10.3916/C49-2016-05

Rivero, P. (2011). Un estudio sobre la efectividad de la multimedia expositiva para el aprendizaje de la Historia. Enseñanza de las Ciencias Sociales. Revista de investigación, $10,45-50$.

Rodríguez, R.A. y Solé, G. (2018). Los manuales escolares de historia en España y Portugal. Reflexiones sobre su uso en Educación Primaria y Secundaria. Arbor: Ciencia, pensamiento y cultura, 194(788). doi: 10.3989/arbor.2018.788n2004

Rodríguez-García, A.M., Romero, J.M. y Campos, N. (2018). De nativos digitales a aprendices digitales: la realidad que se esconde en las universidades españolas. En E. López, F. M. Sirignano, E. Vázquez y A. H. Martín (Eds.), Innovaciones e investigaciones universitarias hispano-italianas (pp. 116-132). Sevilla: GEFORAN.

Romero, J.M. y Rodríguez-García, A.M. (2017). Digitalización de recursos patrimoniales mediante códigos QR: los dispositivos digitales móviles para la difusión cultural y artística en educación. En L. Bocanegra y A. García (Eds.), Con la red, en la red: creación, investigación, comunicación cultural y artística en la era de internet (pp. 595612). Granada: Universidad de Granada y Down Hill Publishing.

Romero, R., Castejón, F.J., López, V.M., y Fraile, A. (2017). Evaluación formativa, competencias comunicativas y TIC en la formación del profesorado. Comunicar: Revista científica iberoamericana de comunicación y educación, 25(52), 73-82. doi: 10.3916/C52-2017-07 
Sanger, E., Silverman, S. y Kraybill, A. (2015). Developing a model for Technology-Based Museum School Partnerships. Journal of Museum Education, 40(2), 147-158. doi: 10.1179/1059865015Z.00000000091

Sobrino, D. (2013). El trabajo con blogs en ciencias sociales, geografía e historia. Clío. History and History Teaching, 39.

Valls, R. (2007). Historiografía escolar española: siglos XIX-XXI. Madrid: UNED. 